\title{
Nanoelectrical analysis of single molecules and atomic-scale materials at the solid/liquid interface
}

\author{
Peter Nirmalraj ${ }^{1 \star}$, Damien Thompson ${ }^{2,3}$, Agustín Molina-Ontoria ${ }^{4}$, Marilyne Sousa ${ }^{1}$, Nazario Martín ${ }^{4}$, \\ Bernd Gotsmann ${ }^{1}$ and Heike Riel ${ }^{1}$
}

Evaluating the built-in functionality of nanomaterials under practical conditions is central for their proposed integration as active components in next-generation electronics. Low-dimensional materials from single atoms to molecules have been consistently resolved and manipulated under ultrahigh vacuum at low temperatures. At room temperature, atomic-scale imaging has also been performed by probing materials at the solid/liquid interface. We exploit this electrical interface to develop a robust electronic decoupling platform that provides precise information on molecular energy levels recorded using in situ scanning tunnelling microscopy/spectroscopy with high spatial and energy resolution in a high-density liquid environment. Our experimental findings, supported by $a b$ initio electronic structure calculations and atomic-scale molecular dynamics simulations, reveal direct mapping of single-molecule structure and resonance states at the solid/liquid interface. We further extend this approach to resolve the electronic structure of graphene monolayers at atomic length scales under standard room-temperature operating conditions.

T he elemental properties of carbon nanostructures are environment dependent. Interpreting the synergism between electronically active nanomaterials and their local chemical domain is pivotal to both scientific and technological interests. Scanning probe microscopy operated at cryogenic conditions with functionalized metal tips ${ }^{1,2}$ in combination with inorganic decoupling platform $\mathrm{s}^{3,4}$ is a widely adopted technique to examine the intramolecular structure of organic materials. In addition to probing matter under ultrahigh-vacuum conditions, scanning tunnelling microscopy (STM) operated at the solid/liquid interface ${ }^{5-11}$ has previously been demonstrated to record real-time molecular dynamics $^{7,12-14}$, register ultrafast chemical reactions $s^{8,15}$, probe the structure of supra-molecular architectures ${ }^{16-18}$ and chemical-fieldeffect transistors ${ }^{19}$, and perform spectral analysis of molecules $s^{6,13,20}$ and real-space visualization of biomolecules ${ }^{5}$ at room temperature. It would be highly attractive to capitalize on principal findings from these two research disciplines to probe the precise electronic states in liquids and quantitatively describe the functionality of single molecules and two-dimensional nanostructures. The experimental challenge lies in addressing the electrochemical congestion present at the solid/liquid electrical interface at room temperature, which stems from the high mobility of organic molecules on metals, the dynamics of the solvent that serves as a liquid sheath and the detrimental effects of the metallic platform due to mixing of metal bands with discrete molecular electronic states ${ }^{21}$. A potential strategy is to employ nonpolar, highly viscous liquid sheaths with low affinity towards the substrate and to engineer a spacer layer ${ }^{3,22,23}$ that electrically disconnects the organic material of interest from metal-induced perturbations. The bottom-up construction of the spacer is crucial as it requires an optimal thickness that suppresses dominant interfacial charge transfer whilst channelizing a small population of electrons to tunnel through the remnant barrier to excite the adsorbed organic material.

Here we report real-space imaging of the intrinsic electronic structure of low-dimensional nanostructures adsorbed on a widebandgap organic insulating spacer layer in an electrochemically inert liquid environment at room temperature. The key advance lies in sandwiching the delicate conductive organic analyte between the ultrathin insulating organic membrane, which electronically decouples the material of interest from the metal, and the top-liquid sheath, which protects the molecules adsorbed on the spacer-coated gold and the STM tunnel gap against external contamination. In the case of single molecules, this multicomponent architecture based on the bottom-spacer and top-liquid sheath also serves to reduce single-molecule mobility, as discerned from molecular dynamics simulations. This reduction in the motion of molecules is essential for resolving single-molecular structure and performing consistent spectroscopic analysis. We further show that the organic spacer layer combined with in situ spectroscopy can sense energy-level shifts (energy resolution $\sim 50 \mathrm{meV}$ ) in chemically tailored molecules, which are rationalized using density functional theory (DFT) calculations. Importantly, the organic spacer serves as an intermediate cushioning layer that prevents metal-induced topological defects in single-layer graphene, thus opening a route towards engineering ultraflat two-dimensional nanostructures on metal supports.

\section{Organic spacer/gold interaction in liquids}

$n$ - $\mathrm{C}_{30} \mathrm{H}_{62}$ (solubilized in 1-phenyloctane) spray-deposited on $\mathrm{Au}(111)$ (Methods) at room temperature ${ }^{24}$ forms close-packed two-dimensional lamellar structures ${ }^{25,26}$. The formation of ordered alkyl-organic layers and their lower dielectric constant $(\sim 2)$ in comparison with inorganic spacers $(\mathrm{NaCl}, \varepsilon: \sim 6)$ make them

${ }^{1}$ IBM Research-Zurich, Säumerstrasse 4, 8803 Rüschlikon, Switzerland, ${ }^{2}$ Department of Physics and Energy, University of Limerick, Ireland, ${ }^{3}$ Materials and Surface Science Institute, University of Limerick, Ireland, ${ }^{4}$ Instituto Madrileño de Estudios Avanzados en Nanociencia (IMDEA-Nanociencia), 
excellent electronic decoupling platforms. The organic layers are homogeneous with an overall thickness of $\sim 4 \AA$, confirmed by ex situ high-sensitivity ellipsometry (Supplementary Section 1.1). At the nanoscale the $n-\mathrm{C}_{30} \mathrm{H}_{62}$ units are seen to adsorb in a fully extended conformation with their backbone axis parallel to the underlying $\mathrm{Au}(111)$ substrate, recorded in the liquid-cell set-up (see schematic Fig. 1a) as shown in the in situ STM image (Fig. 1b) recorded in 1-phenyloctane solvent (for spacer-layer-stability tests see Supplementary Section 1.2). The average length of an individual $n-\mathrm{C}_{30} \mathrm{H}_{62}$ unit is $(3.75 \pm 0.05) \mathrm{nm}$, calculated from the in situ STM topographs and is in agreement with previous STM reports on identical systems $s^{25,26}$. The van der Waals binding energy between the alkyl-spacer and $\mathrm{Au}(111)$ calculated from molecular dynamics structures (Fig. 1c, for details see Supplementary Section 8.1) is $(-2.6 \pm 0.3) \mathrm{eV} \mathrm{nm}^{-2}$, which corresponds to $(-119 \pm 14) \mathrm{kcal} \mathrm{mol}^{-1}$, indicating a strong adsorption of the longchain alkanes on $\mathrm{Au}(111)$, in agreement with the highly ordered structures shown by in situ STM (ref. 25). The inset in Fig. 1b is a high-resolution in situ STM snapshot of the $n-\mathrm{C}_{30} \mathrm{H}_{62}$ units in a silicone oil $\left.\left(\left[-\mathrm{Si}\left(\mathrm{CH}_{3}\right)_{2} \mathrm{O}\right)-\right] n\right)$ environment at room temperature, arranged in an energetically favourable flat-lying configuration. The intermolecular spacing is $\sim 0.5 \mathrm{~nm}$ and the packing energy between adjacent alkane molecules is calculated to be $(-49 \pm 6) \mathrm{meV}$ per carbon, based on atomic-scale modelling (Supplementary Section 8.1). This corresponds to $(-729 \pm 89) \mathrm{meV} \mathrm{nm}^{-2}$, indicating a tightly packed monolayer ${ }^{27}$. The in situ STM data confirm that the long-range geometric ordering and intramolecular configuration of the $n-\mathrm{C}_{30} \mathrm{H}_{62}$ spacer layer remains unperturbed at the interface with silicone oil, reflecting the robustness of the organic spacer architecture. The preservation of organic material integrity by silicone oil, its electrochemical inertness (dielectric constant: 2.5) and density $\left(0.93 \mathrm{~g} \mathrm{ml}^{-1}\right.$ at $\left.25^{\circ} \mathrm{C}\right)$, and the minimization of surface contamination stemming from surrounding hydrocarbons and moisture over time (Supplementary Fig. 2d,e) justify the use of silicone oil as a good liquid sheath model system for in situ probing of soft materials and for imaging atomic-scale features of planar surfaces ${ }^{11}$ under a wide working energy range at room temperature ${ }^{28}$.

\section{Tracking $\mathrm{C}_{60}$ dynamics on solid surfaces in silicone oil}

The geometry, intermolecular mechanics and electronic structure of $\mathrm{C}_{60}$ molecular ensembles directly coupled to noble metals in low-density liquids have been discussed at length ${ }^{13,24,29}$. Here, we focus on the structure, dynamics and energetics of single $\mathrm{C}_{60}$ molecules adsorbed on spacer-coated $\mathrm{Au}(111)$ in the high-density liquid silicone oil. Of particular interest is the interaction strengths between $\mathrm{C}_{60}$ and the spacer layer as well as the subtle interplay between the spacer layer and silicone oil in damping molecular surface diffusion. Following the evaporation of 1-phenyloctane solvent, the alkyl-spacer-coated $\mathrm{Au}(111)$ dosed with pristine $\mathrm{C}_{60}$ molecules (99.9\% purity, purchased in powder form from SigmaAldrich and solubilized in tetrahydrofuran) is encapsulated in a liquid cell with silicone oil as schematically represented in Fig. 1a (for $\mathrm{C}_{60}$ solution synthesis see Supplementary Section 2). The largearea in situ STM image (Fig. 1d) shows a random distribution of $\mathrm{C}_{60}$ molecules with no apparent preferential adsorption site on the spacer layer. The $\mathrm{C}_{60}$ molecules reveal the typical caged structure with a diameter of $(6.5 \pm 0.5) \AA$ (measured over $\sim 80$ individual $\mathrm{C}_{60}$ units) close to the hard-sphere diameter value of $7.1 \AA$ for a single $\mathrm{C}_{60}$ molecule ${ }^{30}$. The spread in the measured diameter value originates from the electronic structure disparity present between the adsorbate and substrate ${ }^{30}$, which is markedly large in the case of a high-conductance $\mathrm{C}_{60}$ molecule adsorbed on a near-insulating alkyl-spacer layer. This difference in electronic structure is evident in Fig. $1 \mathrm{~d}$ where the $\mathrm{C}_{60}$ molecules are better resolved than the underlying spacer layer imaged at a common tunnel-current set point. Note it has not been possible to resolve the multicomponent organic architectures in silicone oil medium simultaneously; however, we observed that the underlying alkyl-spacer structure becomes more prominent when imaged independently (generally at higher negative sample bias and tunnelcurrent set points) revealing sub-molecular image contrast (Fig. 1b, inset). The variations in the local composition of the spacer layer probably originating from the corrugations in the underlying metallic surface are captured in the in situ STM snapshot (Fig. 1d, regions colour coded in green and yellow).

Atomic-scale simulations of film formation support the in situ STM data, indicating that the $\mathrm{C}_{60}$ molecules adsorb directly on the spacer-coated $\mathrm{Au}(111)$. We employ a modified gold van der Waals potential ${ }^{31}$ and model the assembly of the spacer layer from a starting structure of clusters of $n-\mathrm{C}_{30} \mathrm{H}_{62}$ molecules suspended over a bare $\mathrm{Au}(111)$ surface (for details see Supplementary Section 8.2). There is negligible deviation in molecule adsorption energies $(-16 \pm 1) \mathrm{kcal} \mathrm{mol}^{-1}$ for $\mathrm{C}_{60}$ adsorbed at different sites on the spacer layer, with molecule methylene and methyl regions showing comparable, weak van der Waals attraction for $\mathrm{C}_{60}$. The computed structures indicate that the spacer layer is sufficiently stable to prevent the exchange of alkanes for $\mathrm{C}_{60}$ on gold (Supplementary Section 8.2). Taken together, the in situ STM electronic imaging and atomic-scale modelling data show that the spacer layer separates the $\mathrm{C}_{60}$ molecule at an ideal distance from $\mathrm{Au}(111)$, which suppresses direct charge transfer from the metal reservoir but allows a small population of tunnelling electrons.

Interestingly, the isolated $\mathrm{C}_{60}$ molecules as shown in the spatially magnified three-dimensionally represented in situ STM image (Fig. 1e) were stationary and in a kinetically near-frozen state. This is typical of $\mathrm{C}_{60}$ behaviour previously observed only under cryogenic temperatures (4-8 K; refs 32,33). Damping of molecular motion at room temperature has been reported previously for single $\mathrm{C}_{60}$ molecules trapped on engineered porous organic templates $^{34}$, graphene Moiré superlattices ${ }^{35}$ and even macromolecules ${ }^{36}$ on alkyl-coated surfaces. Imaging a single molecule under different positive biasing conditions with a fixed tunnel-current set point exhibited only a bias-dependent profile variation (Fig. 1f,g). This is reminiscent of an electronic effect but reveals no traces of submolecular structure. In control experiments, a $\mathrm{C}_{60}$ molecule placed in direct contact with $\mathrm{Au}(111)$ in a silicone oil environment was imaged (Supplementary Fig. 3b). The structure of the $\mathrm{C}_{60}$ molecules remains unchanged but a comparable drop in mobility was observed that contradicts previous in situ STM observations of $\mathrm{C}_{60}$ dynamics on defect-free $\mathrm{Au}(111)$ (ref. 24) in low-density solvents $(n \text {-tetradecane })^{13}$, where the molecules exhibited high mobility at room temperature. To resolve this puzzling finding of reduced molecular mobility in silicone oil, we rely on molecular dynamics simulations of neat silicone oil (described in detail in Supplementary Section 8.3) that highlight the rigidity of the silicone oil, with a low root mean square fluctuation of $(1.2 \pm 0.3) \AA$, computed in the atomic positions, and a large favourable intermolecular packing energy of $(-125.6 \pm 4.3) \mathrm{kcal} \mathrm{mol}^{-1}$. The computed silicone oil structures (Supplementary Fig. 20) show that the individual molecular strands are highly intertwined through van der Waals contacts and the presence of this top layer of silicone oil (Fig. 1h) reduces the computed mobility of the $\mathrm{C}_{60}$ on both spacer-coated and bare gold (for details see Supplementary Section 8.5). The in situ STM observations and molecular dynamics simulations of $\mathrm{C}_{60}$ dynamics on bare and spacer-coated gold and previous reports of $\mathrm{C}_{60}$ dynamics on gold in low-density liquids ${ }^{13,24}$ confirm that silicone oil plays a dominant role in reducing molecular motion.

\section{In situ mapping of $\mathrm{C}_{60}$ molecular energy levels}

Dielectric spacers are known to weaken metal/molecule interaction energies and thus preserve free-molecular structure $e^{3,37,38}$ recorded by 


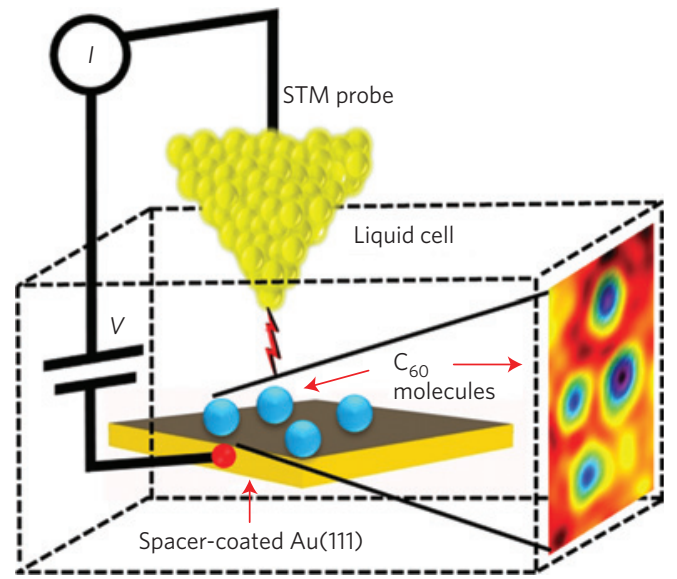

b

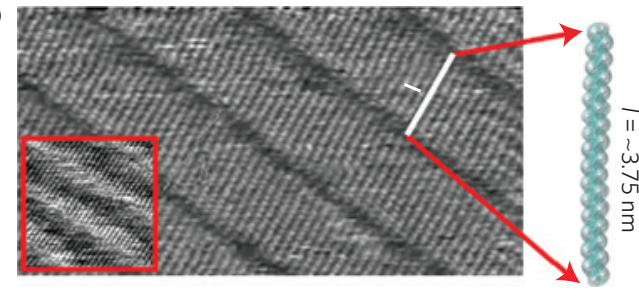

d

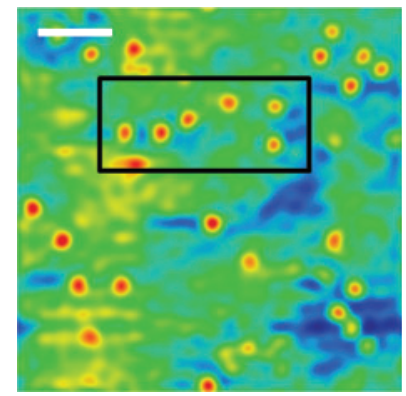

e
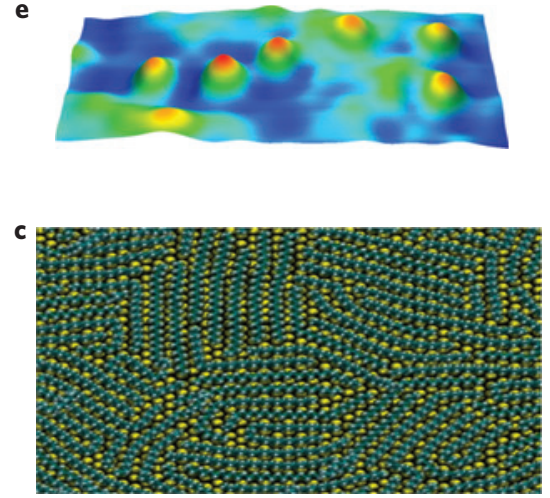

$\mathbf{f}$

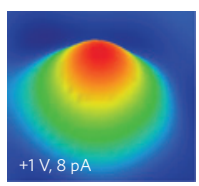

g

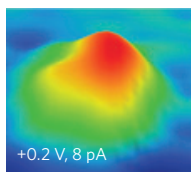

${ }_{\text {Lo }}^{H i}$

h

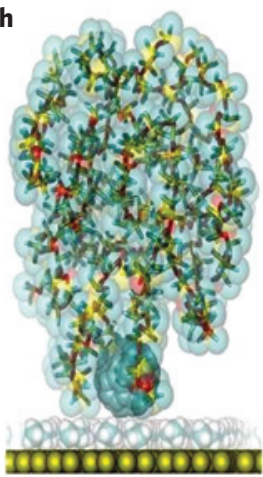

Figure 1 | $\mathbf{C}_{60}$ dynamics on organic spacer-coated $\mathbf{A u}(111)$ in silicone oil. a, Schematic representation of the in situ STM/STS set-up at room temperature, with a representative in situ STM image of the $\mathrm{C}_{60}$ molecules adsorbed on spacer-coated $\mathrm{Au}(111)$ in a silicone oil environment. $\mathbf{b}$, Large-area in situ STM image of the tightly packed alkyl-spacer layer $\left(n-\mathrm{C}_{30} \mathrm{H}_{62}\right.$ monolayer) on $\mathrm{Au}(111)$ imaged in 1-phenyloctane liquid medium (tunnelling set point: I=250 pA, $V=-0.8 \mathrm{mV}$, scan area $25.1 \mathrm{~nm} \times 12.2 \mathrm{~nm}$ ). The white line represents one molecular length as shown in the molecular model of an individual $n-\mathrm{C}_{30} \mathrm{H}_{62}$ unit; the length is $\sim 3.75 \mathrm{~nm}$, calculated from in situ STM images. The inset shows a high-resolution in situ STM image of an $n-\mathrm{C}_{30} \mathrm{H}_{62}$ monolayer resolved under silicone oil medium (tunnelling set point: $I=250 \mathrm{pA}, V=-1.6 \mathrm{~V}$, scan area $11.8 \mathrm{~nm} \times 11.8 \mathrm{~nm}$ ). c, Atom-scale modelling of the initial stage of $n-\mathrm{C}_{30} \mathrm{H}_{62}$ monolayer formation on $\mathrm{Au}(111)$. d, Wide-angle in situ STM image of randomly distributed $\mathrm{C}_{60}$ molecules adsorbed on the organic spacer-coated $\mathrm{Au}(111)$ surface in a silicone oil medium (tunnelling set point: $I=15 \mathrm{pA}, V=0.7 \mathrm{~V}$, scale bar, $3 \mathrm{~nm}$ ). e, Three-dimensionally represented in situ STM of isolated $\mathrm{C}_{60}$ molecules (imaged within a region indicated by the black rectangle in $\mathbf{d}$ ) showing the $\mathrm{C}_{60}$ molecules in a near-frozen state. $\mathbf{f}, \mathbf{g}$, Spatially magnified three-dimensional in situ STM topographs of an individual $\mathrm{C}_{60}$ molecule imaged under different biasing conditions at fixed tunnel-current set points. h, Representative structure calculated for pristine $\mathrm{C}_{60}$ atoms drawn as solid spheres adsorbed on the alkyl-spacer layer (transparent spheres) in the presence of silicone oil molecules (sticks with overlaid transparent spheres; solvent $\mathrm{H}$ atoms omitted for clarity).

ultrahigh-vacuum-based STM/scanning tunnelling spectroscopy (STS). However, mapping electronically decoupled organic electronic structures at the solid/liquid interface at room temperature has not been demonstrated with high spatial and energy resolution until now. We focus here on the electronic influence exerted by the spacer layer on the $\mathrm{C}_{60}$ energy states in liquids using local-point probe in situ STS. Several tips were tested during the STS measurements under silicone oil to confirm reproducibility of the spectral data. To record the spectral data directly, the STM tip is positioned above a well-resolved individual $\mathrm{C}_{60}$ unit (as shown in Fig. 1f) and the voltage is swept with the feedback loop shut off to eliminate tip-sample height fluctuations during measurements (see Methods for in situ STS parameters). Figure $2 \mathrm{a}$ shows a typical differential conductance $(\mathrm{d} I / \mathrm{d} V)$ spectrum of an individual $\mathrm{C}_{60}$ molecule adsorbed on spacercoated $\mathrm{Au}(111)$ that reflects the energetic positions of the frontier molecular orbitals with a discernible low-conductance gap region. The $\mathrm{d} I / \mathrm{d} V$ spectrum exhibits well-structured molecular resonance peaks centred around $-1.1 \mathrm{eV}$ and $+0.9 \mathrm{eV}$ with respect to the Fermi level, which is in contrast with the weak spectral signal of $\mathrm{C}_{60}$ adsorbed directly on $\mathrm{Au}(111)$ in silicone oil (Supplementary Fig. 3b) and with previous in situ STS reports of $\mathrm{C}_{60}$ adsorbed directly on $\mathrm{Au}(111)$ in an $n$-tetradecane environment ${ }^{13}$. The peak positions correspond to the highest occupied molecular orbital (HOMO)and the lowest unoccupied molecular orbital (LUMO)-derived resonances of the $\mathrm{C}_{60}$ molecule. The observed energetic positions are consistent with previous STS observations where lateral electrostatic interactions between $\mathrm{C}_{60}$ and other organic complexes were used to elevate the $\mathrm{C}_{60}$ molecule locally above the $\mathrm{Au}(111)$ surface ${ }^{38}$. In particular, the localization of the LUMO-derived peak at $\sim 0.9 \mathrm{eV}$ above the Fermi edge tends to dominate the charge transport in pristine $\mathrm{C}_{60}$ (refs 39,40), which confirms minimal charge transfer at the metal/molecule electrical interface $e^{41,42}$ as a result of the spacer layer.

The overall spectral line shape of the $\mathrm{d} I / \mathrm{d} V$ spectrum recorded on a specific $\mathrm{C}_{60}$ molecule reveals no significant variation, but slight fluctuations in the molecular-orbital-associated peak positions were observed as measured on several individual $C_{60}$ molecules. We attribute this fluctuation to two key reasons: variation in the tunnelling conditions between each measured molecule in silicone oil; and spacer-layer-induced alterations in the binding geometry and molecular orientation. To map the spread in the molecular orbital peak positions quantitatively we performed a series of in situ STS measurements over $\sim 80$ individual $\mathrm{C}_{60}$ molecules adsorbed on the spacer layer. We derived a conductance gap (energy difference between the HOMO- and LUMO-derived molecular resonance peaks) of $(2.2 \pm 0.3) \mathrm{eV}$ at room temperature, which is close to the previously reported $\Delta E$ value of $2.6 \mathrm{eV}$ where the coupling between $\mathrm{C}_{60}$ and the underlying metal was reduced ${ }^{38}$. It also compares reasonably well to our DFT calculations that predict a $\Delta E$ value of $2.8 \mathrm{eV}$ for a single $\mathrm{C}_{60}$ molecule. The calculated frontier molecular orbital wavefunctions are shown in Fig. $2 \mathrm{~b}$ and the associated experimentally 
a

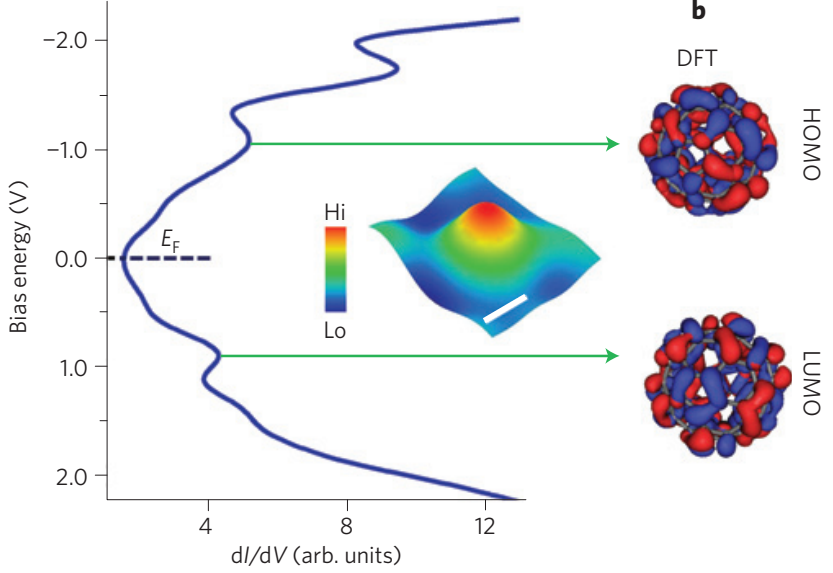

Figure 2 | $\mathrm{C}_{60}$ energy levels on $\boldsymbol{n}$ - $\mathrm{C}_{30} \mathrm{H}_{62}$-coated $\mathrm{Au}(111)$. a, Differential conductance $(\mathrm{d} l / \mathrm{d} V)$ spectrum of the $\mathrm{C}_{60}$ molecules adsorbed on organic spacer-coated $\mathrm{Au}(111)$ in silicone oil medium revealing well-resolved frontier molecular orbitals (feedback loop parameters $I=100 \mathrm{pA}$, $V=1.2 \mathrm{~V}$ ). Inset shows a three-dimensional in situ STM image of a single $\mathrm{C}_{60}$ molecule over which the $\mathrm{d} / \mathrm{d} V$ spectra were recorded (tunnelling set point $I=8 \mathrm{pA}, V=0.4 \mathrm{~V}$, scale bar, $0.6 \mathrm{~nm}$ ). b, Corresponding DFT-calculated highest occupied molecular orbital (HOMO) and lowest unoccupied molecular orbital (LUMO) of the gas-phase $\mathrm{C}_{60}$ molecule.

observed peak positions are indicated by the green arrows (details of the DFT calculations are given in Supplementary Section 8).

\section{Detecting energy-level shifts in chemically modified $\mathrm{C}_{60}$}

To determine whether the proposed metal/molecule weakening mechanism applies beyond pristine molecules, we performed additional experiments on chemically modified $\mathrm{C}_{60}$ molecules. The interest in functionalizing pristine $\mathrm{C}_{60}$ molecules with molecular moieties is driven by the potential application of the $\mathrm{C}_{60}$ cage to act as a stable chemical anchor ${ }^{43,44}$ on the contact metal. This stability of the anchor group ensures well-defined molecular conductance ${ }^{45}$, a germane feature in engineering single-molecule circuits. Of particular interest is the electronic coupling between the $\mathrm{C}_{60}$ anchor and the chemically linked molecular moiety. $\mathrm{C}_{60}$ molecules functionalized with exTTF- $p$-cyclophane (chemical structure shown in Fig. 3a) were deposited from solution (see Supplementary Section 4 for full details on the synthesis and characterization of the functionalized $\mathrm{C}_{60}$ molecules) onto spacer-coated $\mathrm{Au}(111)$ by the same procedure used for pristine $\mathrm{C}_{60}$. The functionalized $\mathrm{C}_{60}$ molecules (hard-sphere diameter $\sim 0.68 \mathrm{~nm}$ ) resolved with nanometre-scale spatial resolution (in situ STM image, Fig. 3c, inset) showed no preferential adsorption site on the spacer layer but exhibited similar damping in molecular motion in the silicone oil medium as in the case of the pristine $\mathrm{C}_{60}$ molecules. Molecular dynamics simulations indicate a reduction in mean square deviation value from 0.25 to $0.15 \mathrm{~nm}^{2}$ for functionalized $\mathrm{C}_{60}$ adsorbed on spacer-coated $\mathrm{Au}(111)$ in the presence of silicone oil (computed structure shown in Fig. 3b). The molecular dynamics calculations provide a quantitative description of functionalized $\mathrm{C}_{60}$ mobility on spacer-coated $\mathrm{Au}(111)$ and substantiate the in situ STM observations. Molecular dynamics models were used to calculate the physisorption energies between the functionalized $\mathrm{C}_{60}$ molecules and the spacer layer, thus providing a deeper understanding of the adsorbate/substrate interfacial interactions. A physisorption energy of $(-27 \pm 4) \mathrm{kcal} \mathrm{mol}^{-1}$ is calculated for functionalized $\mathrm{C}_{60}$ on spacer-coated $\mathrm{Au}(111)$, which is greater than the $(-16 \pm 1) \mathrm{kcal} \mathrm{mol}^{-1}$ calculated for pristine $\mathrm{C}_{60}$. This confirms the participation of the functional groups in the physisorption on the spacer layer (Fig. 3b). Simulations reveal an energetic penalty of $(24 \pm 9) \mathrm{kcal} \mathrm{mol}^{-1}$ for penetration of the functionalized
$\mathrm{C}_{60}$ through the spacer layer and direct adsorption on bare gold (for details see Supplementary Section 8.4), indicating that the molecules will prefer to physisorb directly on the spacer layer and remain decoupled from gold.

The spectroscopic measurements were performed only on wellspaced functionalized $\mathrm{C}_{60}$ molecules (Fig. $3 \mathrm{c}$, inset), to eliminate parallel conduction pathways occurring through the free end of the anchored molecular moiety. Figure $3 \mathrm{c}$ shows a representative $\mathrm{d} I / \mathrm{d} V$ spectral signal acquired over a single functionalized $\mathrm{C}_{60}$ molecule. Two distinct molecular resonance peaks in an energy span of $\pm 1.5 \mathrm{~V}$ are visible in the $\mathrm{d} I / \mathrm{d} V$ spectra centred around -0.5 and $+0.7 \mathrm{eV}$, which correspond to the HOMO and LUMO peak positions, respectively. The DFT-calculated HOMO and LUMO eigenstates in the gas-phase molecule (indicated by the green arrow) show the HOMO localized at the interface between the $\mathrm{C}_{60}$ anchor and the functional group whereas the LUMO is localized on the $\mathrm{C}_{60}$ lobe, comparable to previous DFT calculations on $\mathrm{C}_{60}$-terminated molecular dumbbells ${ }^{39}$, in which the LUMO remains unperturbed on molecular functionalization. Also the LUMO-derived peak position for the functionalized $\mathrm{C}_{60}$ molecule with respect to the Fermi edge is equivalent to the LUMO position of pristine $\mathrm{C}_{60}$ but there is a significant shift in the HOMO peak position closer to the Fermi edge resulting in a decreased conductance gap for the functionalized $\mathrm{C}_{60}$ molecule. Having measured more than $\sim 65$ isolated functionalized $\mathrm{C}_{60}$ molecules, we derived a $\Delta E$ value of $(1.1 \pm 0.2) \mathrm{eV}$, which is close to our DFTcalculated $\Delta E$ value of $0.9 \mathrm{eV}$ for a functionalized $\mathrm{C}_{60}$ molecule. The observed reduction in $\Delta E$ for a functionalized $\mathrm{C}_{60}$ molecule in comparison with pristine $\mathrm{C}_{60}(\Delta E=2.2 \pm 0.3 \mathrm{eV}$, above $)$ suggests good electrical coupling between the anchor $\left(\mathrm{C}_{60}\right)$ and the core of the chemically linked molecular moiety (exTTF-p-cyclophane) with minimal functionalization-induced symmetry disruption to the $\mathrm{C}_{60}$ cage. This ability to detect and discern the origin of the electronic shifts on $\mathrm{C}_{60}$-anchor-based molecular complexes in a selective manner is possible only by weakening the tendency of the anchor $\left(\mathrm{C}_{60}\right)$ molecular orbitals to hybridize with gold ${ }^{43}$. If allowed to occur, this hybridization results in significant broadening and clouding of intrinsic spectral features (see Supplementary Fig. 12b for in situ STS measurements of functionalized $\mathrm{C}_{60}$ on bare gold).

\section{Atom-scale analysis of graphene in silicone oil}

Tuning $\mathrm{metal} / \mathrm{molecule}$ electronic interactions by inserting insulating organic spacers at the metal/molecule interface is not limited to single molecules. In addition we can electronically decouple singlelayer graphene from metals and record its unique band structure in liquids. Liquid-phase-exfoliated graphene (see Supplementary Section 6 for sample preparation) in low-boiling-point solvents was spray-deposited onto spacer-coated Au (111) and placed directly in a liquid cell packed with silicone oil. The single-layer graphene flakes possess well-defined edges with a lateral flake size ranging from 0.2 to $1.0 \mu \mathrm{m}$ and a characteristic thickness of $0.35-0.5 \mathrm{~nm}$ based on in situ STM and intermittent-contact atomic force microscopy $^{46}$ (AFM; Methods). The atomic plane of carbon atoms of graphene is evident from the high-resolution constant-current in situ STM image (Fig. 4a). The graphene crystal structure can be distinctly imaged in silicone oil at room temperature with a resolution on par with graphene resolved using cryogenic STM (refs 47,48). The hexagonal lattice of carbon atoms can also be resolved at high tunnel-current set points $(\sim 1.8 \mathrm{nA})$, reflecting the structural stability of the interface between the graphene and the spacer layer, previously observed for graphene on two-dimensional insulating platforms ${ }^{47}$. Molecular dynamics simulations (Supplementary Section 8.6) revealed a binding energy of -1.0 and $-3.8 \mathrm{eV} \mathrm{nm}^{-2}$ for graphene on spacer-coated and bare gold, respectively, in good agreement with dispersion-corrected DFT (ref. 49). Summing over the graphene/spacer and spacer/gold interfaces 


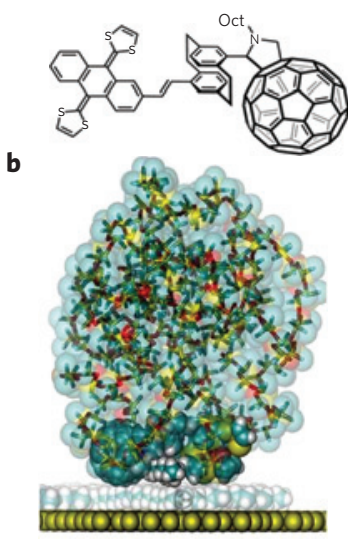

C

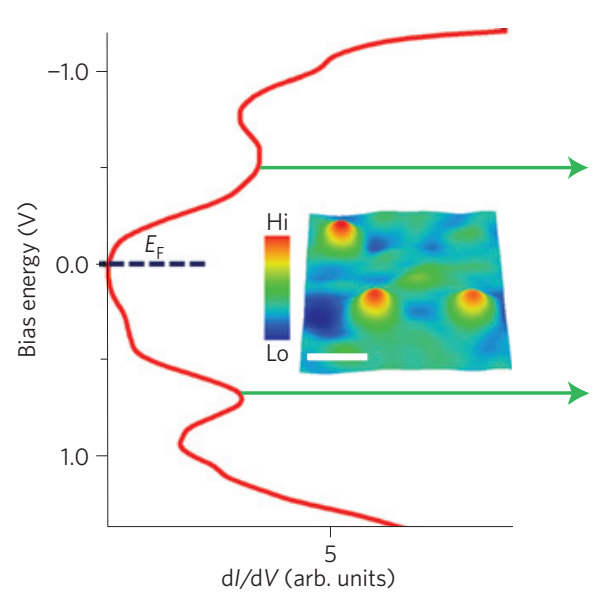

d
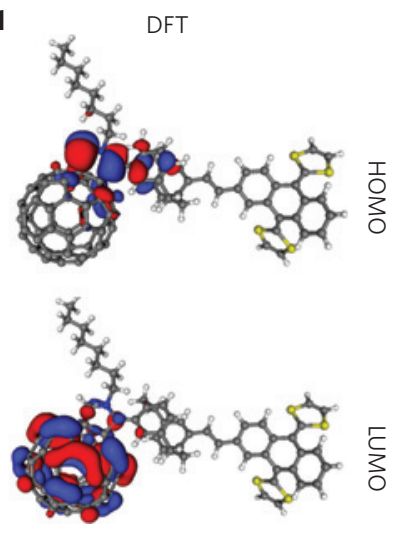

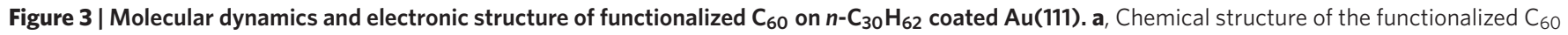
molecule, with an exTTF-p-cyclophane moiety tethered to the $\mathrm{C}_{60}$ cage. $\mathbf{b}$, A representative snapshot from molecular dynamics simulations of

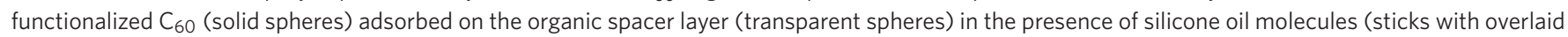

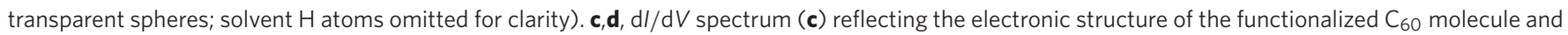
the associated DFT-calculated frontier molecular orbitals (d) in the gas-phase molecule indicated by green arrows (feedback loop parameters $I=100$ pA, $V=1.2 \mathrm{~V}$ ). The inset in c shows a three-dimensionally depicted in situ STM image of the functionalized $\mathrm{C}_{60}$ molecules adsorbed on an alkyl-spacer-coated $\mathrm{Au}(111)$ surface in a silicone oil medium $(I=5 \mathrm{pA}, V=0.6 \mathrm{~V}$, scale bar, $1 \mathrm{~nm})$

yields a net energy of $-4.3 \mathrm{eV} \mathrm{nm}^{-2}$, indicating a preference of $\sim 500 \mathrm{meV} \mathrm{nm}^{-2}$ for graphene adsorption on the spacer layer rather than on bare gold. This implies that a single layer of graphene can lie on the spacer layer without disrupting its geometric order (see Supplementary Section 8.6 for binding-energy calculations). Largearea in situ STM images show that the overall graphene landscape does not suffer from a high density with topological defects (Supplementary Fig. 14a) when compared to single-layer graphene placed directly in contact with $\mathrm{Au}(111)$ as shown in Fig. 4c, which reveals regions where the lattice symmetry is clearly disrupted in addition to the presence of local-point defects on the atomic lattice consistent with previous reports of graphene adsorbed on noble metals ${ }^{48,50}$.

The electronic disentanglement of graphene from the metal through spacer-layer mediation was verified by in situ STS measurements. We then compared the spectral signatures between graphene adsorbed on the spacer layer and graphene directly in contact with $\mathrm{Au}(111)$. Figure $4 \mathrm{~b}$ shows a well-resolved $\mathrm{d} I / \mathrm{d} V$ spectrum (acquired over the sites indicated in the in situ STM image, Fig. 4a) with a distinctive line shape resembling the energy spectrum of graphene, similar to previous STS observations of graphene on non-metallic substrates ${ }^{51-53}$. The minimum in each of the directly measured spectral curves corresponds to the charge-neutral Dirac point $\left(E_{\mathrm{D}}\right)$ located at positive bias energy slightly above the Fermi level. To quantify the spatial variation of the energy landscape, $\mathrm{d} I / \mathrm{d} V$ spectra were acquired over a monolayer graphene area of $100 \mathrm{~nm}^{2}$ under identical tunnelling conditions, which yields a mean $E_{\mathrm{D}}$ of $(48 \pm 14) \mathrm{mV}$ (Dirac point shift histogram; Fig. 4b, inset) indicative of minimal substrate-induced doping. The spectral data for graphene on spacer-coated gold are remarkably different from the spectral signature for graphene on bare gold (Supplementary Fig. 15) where two significant dips at $E_{\mathrm{F}}$ and $E_{\mathrm{D}}$ now located at $\sim+350 \mathrm{mV}$ are discernible. This observation of $E_{\mathrm{D}}$ occurring at higher positive bias energy $(\sim+350 \mathrm{mV})$, with respect to the Fermi edge, clearly points to alignment of the Fermi levels occurring through charge transfer between the gold surface and graphene. This shift in $E_{\mathrm{D}}$ is comparable to previous analytical modelling reports of metal doping on graphene ${ }^{54}$ and low-temperature STS (ref. 48) observations of graphene in contact with metals.

In addition to mapping the variations in electronic structure by detecting the shifts in $E_{\mathrm{D}}$ for graphene, the bonding configuration of in-plane atoms in graphene can be clearly resolved in liquids at room-temperature imaging conditions. The atomic positions of the $s p^{2}$-bonded carbon atoms and the respective bond lengths can be precisely identified on the basis of high-resolution in situ STM images. Figure $4 \mathrm{~d}$ shows a three-dimensional in situ STM image of a locally magnified region on single-layer graphene adsorbed on spacer-coated gold imaged under silicone oil. The $\mathrm{C}-\mathrm{C}$ bond distance measured over a well-resolved atomic hexagon as seen in Fig. $4 \mathrm{~d}$ is $\sim 1.42 \AA$. An average $\mathrm{C}-\mathrm{C}$ bond distance (measured over all atomic hexagons over an area of $100 \mathrm{~nm}^{2}$ of single-layer graphene) yields a value of $(1.38 \pm 0.12) \AA$. Imaging over the same graphene lattice region (Fig. 4d) and incrementing the bias in small steps revealed that the local electronic contrast over the atomic hexagons is tunable and is dependent on the energy of the tunnelling electrons (Fig. 4e). These observations highlight that useful information from bond length fluctuations to atomic-scale contrast can be extracted from two-dimensional materials in a liquid medium at room temperature. Resolving in real-space the atomic positions in graphene immersed in liquids using a non-functionalized STM probe reflects the versatility of this technique. Importantly, the inherent nature of silicone oil, with its rigid molecular packing structure and electrochemical inertness, provides the necessary high stability during imaging at room temperature by minimizing liquidinduced thermal fluctuations, preventing tip-apex contamination and parasitic faradaic current at the STM junction.

\section{Outlook}

We provide compelling evidence for the singular read-out of integral mono-molecular and atomic-material electronic structure on an insulating organic layer using in situ STM and STS. The engineering of an organic membrane with ångström-scale thickness control, scalable to industrial needs, can be extended to printed electronics where a percolating organic nanomaterial network is requisite. The findings deepen our understanding on the dependence of molecular electronic states of pristine and chemically tailored carbon particles on their interfacial structure in complex environments, thus suggesting a reliable route towards chemical sensing of complex analytes. It is expected that the spatial resolution capability can be further advanced by chemically terminating the scanning probe tip-apex ${ }^{1,2,23,55}$. We draw particular attention to the potential of 


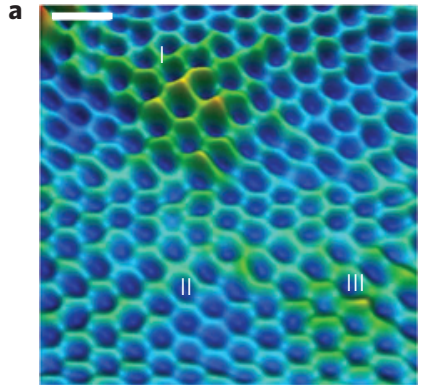

Lo $\mathrm{Hi}$

d

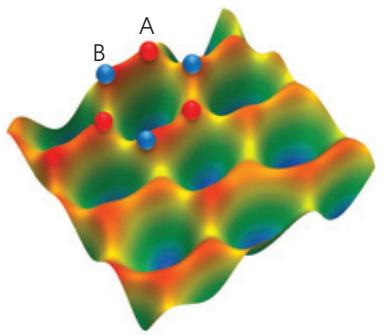

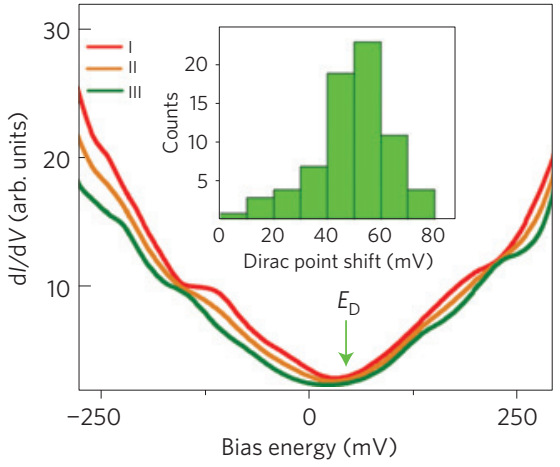

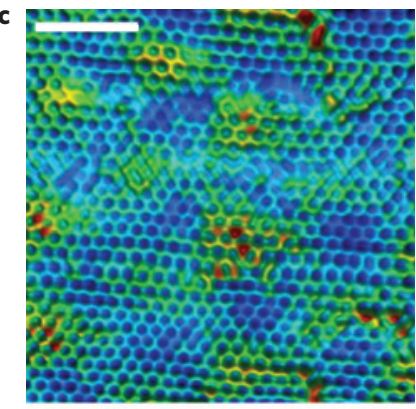

Lo $\mathrm{Hi}$
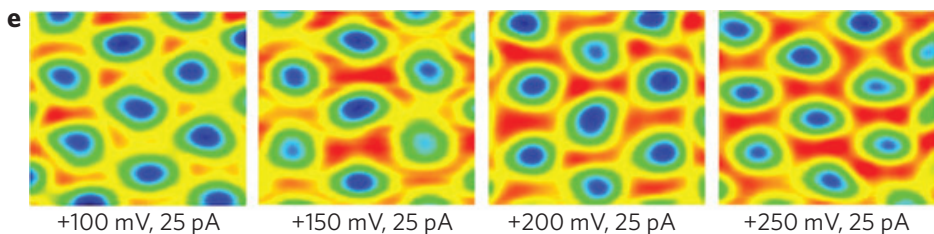

Lo $\mathrm{Hi}$

Figure 4 | Atom-scale mapping of graphene electronic structure. a, High-resolution three-dimensional in situ STM topographic image of a graphene monolayer adsorbed on the organic spacer-coated $A u(111)$ surface $(I=15 \mathrm{pA}, V=0.8 \mathrm{~V}$, scale bar, $2.2 \AA)$. b, $d / / d V$ spectral curves acquired over the locations indicated in a revealing a characteristic graphene linear band structure. The green arrow indicates the Dirac point ( $E_{\mathrm{D}}$; feedback loop parameters $I=150 \mathrm{pA}, \mathrm{V}=1.5 \mathrm{~V})$. The inset shows the histogram of fluctuations in the measured Dirac point over a graphene area of $100 \mathrm{~nm}^{2} . \mathbf{c}, W i d e-a n g l e ~ i n ~ s i t u$ STM image of topological defects on a monatomic graphene adsorbed directly on Au(111) in silicone oil environment ( $I=40 \mathrm{pA}, V=1 \mathrm{~V}, \mathrm{scale}$ bar, $2 \mathrm{~nm})$. d, High-resolution in situ STM of graphene on spacer-coated Au(111) where the atomic positions are well resolved (scan area: $0.75 \mathrm{~nm} \times 0.8 \mathrm{~nm}$ ). The red and blue spheres marked on the graphene lattice represent the two atoms per unit cell depicted as A and B. e, Bias-dependent imaging of the graphene atomic hexagons in silicone oil (scan area: $0.75 \mathrm{~nm} \times 0.8 \mathrm{~nm}$ ). The in situ STM/STS measurements on monolayer graphene adsorbed on the spacer-coated and bare gold were performed in a silicone oil environment at room temperature.

silicone oil as liquid brakes for damping single-molecular motion that provides a stable platform for nanoelectrical measurements at the solid/liquid interface. This approach of reducing the surface dynamics of molecules at room temperature using high-density liquids is central for probing energy levels of biomolecules such as nucleic acids interfacing with graphene, intriguing systems that remain to be fully understood.

\section{Methods}

Preparation of gold substrate. One hundred nanometres of $\mathrm{Au}(99.99 \%$, FHR Anlagenbau GmbH) was sputter-deposited (Cluster system CS320S, Von Ardenne, Dresden) in an argon atmosphere onto $\mathrm{CaF}_{2}(111)$ substrates $\left(2 \mathrm{~cm} \times 2 \mathrm{~cm}\right.$, pre-heated at $500^{\circ} \mathrm{C}$ for $\left.3 \mathrm{~h}\right)$ at a rate of $10 \AA \mathrm{s}^{-1}$ and a base pressure of $2 \times 10^{-7} \mathrm{mbar}$. The dominant (111) texture for the resulting metal films was confirmed using high-resolution X-ray diffraction.

Spacer-layer fabrication. $n-\mathrm{C}_{30} \mathrm{H}_{62}$ (Triacontane, 98\%) was purchased from Sigma-Aldrich and used without any additional purification procedure. A near-saturated solution of this compound was prepared in 1-phenyloctane solvent (Sigma-Aldrich) and subjected to gentle bath sonication for 1 min. Half a millilitre of this solution was spray-coated (Dual action, Evolution Airbrush, Harder Steenbeck, nozzle size, $0.15 \mathrm{~mm}$ ) using a single pass onto freshly prepared $\mathrm{Au}(111)$ substrates and immediately placed in the liquid-cell chamber for in situ STM imaging.

In situ STM/STS and AFM measurements. STM measurements were performed in constant-current mode using a Digital Instruments Nanoscope IIIa scanning tunnelling microscope (Multimode System, scanner model: E-Scanner). Nanoscale calibration of the scanner was performed using a freshly cleaved highly oriented pyrolytic graphite surface ( $x, y$ axes) and a $\mathrm{Au}(111)$ on mica substrate ( $z$ axis). Bias voltages imply sample voltage with respect to the STM probe. For the STM probe, mechanically cut Au wire $(0.25 \mathrm{~mm}$, Good Fellow $\mathrm{GmbH})$ was used. The quality of the tip was constantly monitored by imaging and acquiring $\mathrm{d} I / \mathrm{d} V$ spectra over clean $\mathrm{Au}(111)$-mica samples as reference. Low tunnel-current set points were employed to avoid tip-induced motion of the single molecules. For the in situ STS measurements the feedback loop is turned off $(\sim 30 \mathrm{~s})$ after ensuring the tip is positioned above the molecule of interest and the voltage is swept while the current is recorded. The tip drift rate is $\leq 1 \mathrm{~nm} \mathrm{~min}{ }^{-1}$ and it takes $\sim 2 \mathrm{~s}$ to record a single spectral curve. Each $\mathrm{d} I / \mathrm{d} V$ spectrum thus recorded (on individual and non-interconnected pristine fullerene and functionalized fullerene molecules) was averaged over 20 voltage sweeps. To check the reproducibility of the differential conductance spectra reported for the single molecules we thoroughly analyse each forward and reverse voltage sweep (tip stability and image resolution is constantly monitored) for each individual case and the molecular structure was verified before and after acquisition of the spectral curve. A custom-built liquid-cell holder was used for in situ STM and STS measurements. AFM measurements were performed on the same system with an interchangeable optical head in intermittent-contact mode using $n$-type silicon tips with aluminium coating on the detector side (Nanosensors, 200-500 kHz resonant frequency). The in situ STM/STS set-up is located in a noise-free laboratory (Supplementary Section 3).

Received 11 October 2013; accepted 15 July 2014; published online 17 August 2014

\section{References}

1. De Oteyza, D. G. et al. Direct imaging of covalent bond structure in single-molecule chemical reactions. Science 340, 1434-1437 (2013).

2. Gross, L., Mohn, F., Moll, N., Liljeroth, P. \& Meyer, G. The chemical structure of a molecule resolved by atomic force microscopy. Science 325, 1110-1114 (2009).

3. Repp, J., Meyer, G., Stojkovic, S. M., Gourdon, A. \& Joachim, C. Molecules on insulating films: Scanning-tunneling microscopy imaging of individual molecular orbitals. Phys. Rev. Lett. 94, 026803 (2005).

4. Shin, H. J. et al. State-selective dissociation of a single water molecule on an ultrathin $\mathrm{MgO}$ film. Nature Mater. 9, 442-447 (2010).

5. Rabe, J. P. Molecules at interfaces: STM in materials and life sciences. Ultramicroscopy 42-44, 41-54 (1992).

6. Uji, I. H. et al. Scanning tunneling microscopy and spectroscopy of donor-acceptor-donor triads at the liquid/solid interface. ChemPhysChem 6, 2389-2395 (2005)

7. De Feyter, S. \& De Schryver, F. C. Self-assembly at the liquid/solid interface: STM reveals. J. Phys. Chem. 109, 4290-4302 (2005). 
8. Hulsken, B. et al. Real-time single-molecule imaging of oxidation catalysis at a liquid-solid interface. Nature Nanotech. 2, 285-289 (2007).

9. Sonnenfeld, R. \& Hansma, P. K. Atomic-resolution microscopy in water. Science 232, 211-213 (1986).

10. Itaya, K., Sugawara, S. \& Higaki, K. In situ scanning tunneling microscopy for platinum surfaces in aqueous solutions. J. Phys. Chem. 92, 6714-6718 (1988).

11. Giambattista, B. et al. Atomic resolution images of solid-liquid interfaces. Proc. Natl Acad. Sci. USA 84, 4671-4674 (1987).

12. De Feyter, S., Xu, H. \& Mali, K. Dynamics in self-assembled organic monolayers at the liquid/solid interface revealed by scanning tunneling microscopy. Chimia 66, 38-43 (2012).

13. Katsonis, N., Marchenko, A. \& Fichou, D. Dynamics and spectroscopy of single C60 molecules adsorbed on $\mathrm{Au}(111)$ at the liquid-solid interface. J. Photochem. Photobiol. A 158, 101-104 (2003).

14. Rabe, J. P. \& Buchholz, S. Direct observation of molecular structure and dynamics at the interface between a solid wall and an organic solution by scanning tunneling microscopy. Phys. Rev. Lett. 66, 2096-2099 (1991).

15. Den Boer, D. et al. Detection of different oxidation states of individual manganese porphyrins during their reaction with oxygen at a solid/liquid interface. Nature Chem. 5, 621-627 (2013).

16. Ciesielski, A., Palma, C. A., Bonini, M. \& Samori, P. Towards supramolecular engineering of functional nanomaterials: Pre-programming multi-component 2D self-assembly at solid-liquid interfaces. Adv. Mater. 22, 3506-3520 (2010).

17. Elemans, J. A. A. W. \& De Feyter, S. Structure and function revealed with submolecular resolution at the liquid-solid interface. Soft Matter 5, 721-735 (2009).

18. Samori, P. et al. Supramolecular staircase via self-assembly of disklike molecules at the solid-liquid interface. J. Am. Chem. Soc. 123, 11462-11467 (2001).

19. Jackel, F., Watson, M. D., Mullen, K. \& Rabe, J. P. Prototypical single-molecule chemical-field-effect transistor with nanometer-sized gates. Phys. Rev. Lett. 92, 188303 (2004).

20. Albrecht, T. et al. Scanning tunneling spectroscopy in an ionic liquid. J. Am. Chem. Soc. 128, 6574-6575 (2006).

21. Moth-Poulsen, K. \& Bjornholm, T. Molecular electronics with single molecules in solid-state devices. Nature Nanotech. 4, 551-556 (2009).

22. Gopakumar, T. G. et al. Coverage-driven electronic decoupling of Fe-phthalocyanine from a $\operatorname{Ag}(111)$ substrate. J. Phys. Chem. C 115, 12173-12179 (2011).

23. Gross, L. Recent advances in submolecular resolution with scanning probe microscopy. Nature Chem. 3, 273-278 (2011).

24. Nirmalraj, P. N., Schmid, H., Gotsmann, B. \& Riel, H. Nanoscale origin of defects at metal/molecule engineered interfaces. Langmuir 29, 1340-1345 (2013).

25. Zhang, H-M., Xie, Z-X., Mao, B-W. \& Xu, X. Self-assembly of normal alkanes on the Au (111) surfaces. Chem. Eur. J. 10, 1415-1422 (2004).

26. Marchenko, O. \& Cousty, J. Molecule length-induced reentrant self-organization of alkanes in monolayers adsorbed on Au(111). Phys. Rev. Lett. 84, 5363-5366 (2000).

27. Nerngchamnong, N. et al. The role of van der Waals forces in the performance of molecular diodes. Nature Nanotech. 8, 113-118 (2013).

28. Akari, S. et al. Anomalous voltage dependence of tunnelling microscopy in $\mathrm{WSe}_{2}$. J. Microsc. 152, 521-526 (1988).

29. Marchenko, A. \& Cousty, J. $\mathrm{C}_{60}$ self-organization at the interface between a liquid $\mathrm{C}_{60}$ solution and a $\mathrm{Au}(111)$ surface. Surf. Sci. 513, 233-237 (2002).

30. Weckesser, J., Barth, J. V. \& Kern, K. Mobility and bonding transition of $\mathrm{C}_{60}$ on Pd(110). Phys. Rev. B 64, 161403 (2001).

31. Heinz, H., Vaia, R. A., Farmer, B. L. \& Naik, R. R. Accurate simulation of surfaces and interfaces of face-centered cubic metals using 12-6 and 9-6 Lennard-Jones potentials. J. Phys. Chem. 112, 17281-17290 (2008).

32. Grill, L. Functionalized molecules studied by STM: Motion, switching and reactivity. J. Phys. Condens. Matter 20, 053001 (2008).

33. Neel, N. et al. Controlled contact to a $\mathrm{C}_{60}$ molecule. Phys. Rev. Lett. 98, 065502 (2007).

34. Griessl, S. J. H. et al. Room-temperature scanning tunneling microscopy manipulation of single C60 molecules at the liquid-solid interface: Playing nanosoccer. J. Phys. Chem. B 108, 11556-11560 (2004).

35. Lu, J. et al. Using the graphene Moiré pattern for the trapping of $\mathrm{C}_{60}$ and homoepitaxy of graphene. ACS Nano 6, 944-950 (2011).

36. Nikolai Severin, J. B., Alexey, A. K. \& Jurgen, P. R. Manipulation and overstretching of genes on solid substrates. Nano Lett. 4, 577-579 (2004).
37. Wang, Y., Kroger, J., Berndt, R. \& Tang, H. Molecular nanocrystals on ultrathin $\mathrm{NaCl}$ films on $\mathrm{Au}(111)$. J. Am. Chem. Soc. 132, 12546-12547 (2010).

38. Franke, K. J. et al. Reducing the molecule-substrate coupling in $\mathrm{C}_{60}$-based nanostructures by molecular interactions. Phys. Rev. Lett. 100, 036807 (2008).

39. Bilan, S., Zotti, L. A., Pauly, F. \& Cuevas, J. C. Theoretical study of the charge transport through $\mathrm{C}_{60}$-based single-molecule junctions. Phys. Rev. B 85, 205403 (2012).

40. Néel, N. et al. Controlled contact to a $\mathrm{C}_{60}$ molecule. Phys. Rev. Lett. 98, 065502 (2007).

41. Franke, K. J. \& Pascual, J. I. Effects of electron-vibration coupling in transport through single molecules. J. Phys. Condens. Matter 24, 394002 (2012).

42. Lu, X., Grobis, M., Khoo, K. H., Louie, S. G. \& Crommie, M. F. Charge transfer and screening in individual $\mathrm{C}_{60}$ molecules on metal substrates: A scanning tunneling spectroscopy and theoretical study. Phys. Rev. B 70, 115418 (2004).

43. Martin, C. A. et al. Fullerene-based anchoring groups for molecular electronics. J. Am. Chem. Soc. 130, 13198-13199 (2008).

44. Lortscher, E. et al. Bonding and electronic transport properties of fullerene and fullerene derivatives in break-junction geometries. Small 9, 209-214 (2013).

45. Markussen, T., Settnes, M. \& Thygesen, K. S. Robust conductance of dumbbell molecular junctions with fullerene anchoring groups. J. Chem. Phys. 135, 144104-144106 (2011).

46. Nirmalraj, P. N., Lutz, T., Kumar, S., Duesberg, G. S. \& Boland, J. J. Nanoscale mapping of electrical resistivity and connectivity in graphene strips and networks. Nano Lett. 11, 16-22 (2011).

47. Decker, R. et al. Local electronic properties of graphene on a BN substrate via scanning tunneling microscopy. Nano Lett. 11, 2291-2295 (2011).

48. Ugeda, M. M. et al. Point defects on graphene on metals. Phys. Rev. Lett. 107, 116803 (2011).

49. Zheng, J. et al. Interfacial properties of bilayer and trilayer graphene on metal substrates. Sci. Rep. 3, 2081 (2013).

50. Banhart, F., Kotakoski, J. \& Krasheninnikov, A. V. Structural defects in graphene. ACS Nano 5, 26-41 (2010).

51. Cho, J. et al. Structural and electronic decoupling of $\mathrm{C}_{60}$ from epitaxial graphene on SiC. Nano Lett. 12, 3018-3024 (2012).

52. Luican, A., Li, G. \& Andrei, E. Y. Quantized Landau level spectrum and its density dependence in graphene. Phys. Rev. B 83, 041405 (2011).

53. Miller, D. L. et al. Observing the quantization of zero mass carriers in graphene. Science 324, 924-927 (2009).

54. Giovannetti, G. et al. Doping graphene with metal contacts. Phys. Rev. Lett. 101, 026803 (2008).

55. Schull, G., Frederiksen, T., Brandbyge, M. \& Berndt, R. Passing current through touching molecules. Phys. Rev. Lett. 103, 206803 (2009).

\section{Acknowledgements}

The authors thank R. Stutz and H. Schmid for metal-deposition experiments, M. Tschudy for liquid-cell fabrication and E. Lörtscher for designing the noise-free laboratories. P.N. thanks H. Wolf, F. Schwarz and J. Boland for useful discussions. This work was supported by the Marie Curie Actions-Intra-European Fellowship (IEF-PHY) under grant agreement No 275074 'To Come' within the 7th European Community Framework Programme. D.T. thanks Science Foundation Ireland (SFI) for financial support under Grant Number 11/SIRG/B2111 and the SFI/Higher Education Authority Irish Center for High-End Computing (ICHEC). This work was supported by the EC FP7ITN 'FUNMOLS' Project Number: PITN-GA-2008-212942.

\section{Author contributions}

P.N. designed and performed the in situ STM/STS experiments. M.S. carried out the ellipsometry measurements and data analysis. A.M-O. and N.M. synthesized the functionalized $\mathrm{C}_{60}$ molecules and performed the electrochemical characterization. D.T. designed and performed the molecular dynamics simulations and DFT calculations. All authors contributed and commented on the manuscript and analysis of the data.

\section{Additional information}

Supplementary information is available in the online version of the paper. Reprints and permissions information is available online at www.nature.com/reprints. Correspondence and requests for materials should be addressed to P.N.

\section{Competing financial interests}

The authors declare no competing financial interests. 